\title{
TESTES PSICOLÓGICOS UTILIZADOS PARA AVALIAR ESTRESSE NA CRIANÇA: UMA REVISÃO INTEGRATIVA
}

\author{
Carla Araujo Bastos Teixeira ${ }^{1}$ \\ Erica Taciana dos Santos Crepaldi ${ }^{2}$ \\ Edilaine Cristina da Silva Gherardi-Donato ${ }^{3}$ \\ Emilene Reisdorfer ${ }^{4}$ \\ Ana Maria Pimenta Carvalho ${ }^{5}$ \\ Patrícia Leila dos Santos ${ }^{6}$
}

TEIXEIRA, C. A. B.; CREPALDI, E. T. dos S.; GHERARDI-DONATO, E. C. da S.; REISDORFER, E.; CARVALHO, A. M. P.; SANTOS, P. L. dos. Testes psicológicos utilizados para avaliar estresse na criança: uma revisão integrativa. Arq. Cienc. Saúde UNIPAR, Umuarama, v. 19 , n. 1, p, 53-58, jan./abr. 2015

\begin{abstract}
RESUMO: Realizar uma revisão integrativa da literatura na Biblioteca Virtual em Saúde (BVS) e PubMed buscando responder à pergunta de pesquisa: "Quais são os testes psicológicos utilizados para avaliar estresse na infância?" Na base de dados BVS foram utilizados os descritores "Estresse Psicológico"; "Testes Psicológicos"; "Crianças". Na plataforma PubMed foi realizada pesquisa pelos descritores "Psychological Stress" AND "Psychological Tests" AND "Child”. Em seguida, foram utilizados filtros e critérios de exclusão em ambas as bases (ano de publicação: últimos dez anos; idioma: inglês, português; limite de idade: crianças até 12 anos; tipo de documento: artigo). Após exclusão de artigos duplicados nas respectivas bases, leitura dos títulos, resumos e artigos na íntegra, restaram 11 artigos relacionados ao tema para realizar esta revisão integrativa da literatura. A deficiência de testes psicológicos específicos que avaliem estresse infantil pode apontar a dificuldade de mensurá-lo, pois o estresse é um processo complexo de aferição multicentralizada. Considera-se relevante o desenvolvimento de estudos que elucidem a avaliação do estresse em crianças para que, deste modo, subsidie o desenvolvimento de pesquisas neste campo de estudo.
\end{abstract}

PALAVRAS-CHAVE: Estresse psicológico; Testes psicológicos; Criança.

\section{PSYCHOLOGICAL TESTS USED TO EVALUATE STRESS IN CHILDREN: AN INTEGRATIVE REVIEW}

\begin{abstract}
To perform an integrative review of the literature in the Virtual Health Library (BVS) and PubMed, seeking to answer the research question: "What are the psychological tests used to assess stress in childhood?" In the BVS database, the following descriptors were used: "Psychological Stress"; "Psychological Tests"; "Children". In PubMed platform, the research was carried out by using the descriptors: "Psychological Stress" AND "Psychological Tests" AND "Child". The following filters and exclusion criteria were used in both databases: (year of publication: last ten years; language: English and Portuguese; age limit: children up to 12 years old; type of document: article). After exclusion of duplicate items in the databases, reading the titles, abstracts and full articles, 11 articles related to the topic were selected to be part in this integrative literature review. The deficiency of specific psychological tests to assess childhood stress demonstrates the difficulty of measuring it, since stress is a complex process of multi-centered assessment. There is the need to develop further studies to elucidate the assessment of stress in children in order to subsidize the development of research in this field of study.
\end{abstract}

KEYWORDS: Stress psychological; Psychological tests; Child.

\section{Introdução}

O estresse é uma terminologia utilizada no mundo contemporâneo de forma vasta e seu emprego varia em consonância com o referencial teórico adotado. A literatura apresenta três correntes conceituais principais: biológica, agente externo de mudança, transacional (NAKASATO; CAROMANO, 2000; GHERARDI-DONATO, 2013; TEIXEIRA, 2013).

Selye, (1956), o primeiro pesquisador a empregar este termo no campo da saúde, definiu estresse sob uma perspectiva biológica, entendendo-o como resposta fisiológica do indivíduo, a qual pode ser cronificada dependendo da natureza e duração da fonte estressora. Tal corrente conceitual não considerava as emoções como fatores significativos (SELYE, 1956).
Partindo de outra perspectiva, Holmes e Rahe (1967) conceituaram estresse como agente externo, ou seja, como um acontecimento qualquer causador de mudança no padrão habitual de vida. Essa definição ampliou a conceituação do estresse, mas ainda desconsiderou o fator indivíduo (HOLMES e RAHE, 1967).

Suplantando as correntes anteriores, Lazarus e Folkman (1984) desvelaram o estresse numa perspectiva transacional, analisando-o a partir da interação entre o sujeito e o ambiente, levando em consideração aspectos cognitivos, comportamentais e a percepção do próprio indivíduo sobre a situação estressora (LAZARUS; FOLKMAN, 1984; GHERARDI-DONATO, 2013; TEIXEIRA, 2013). Mais recentemente, Vanaelst (2012) avança nessa perspectiva compreendendo o estresse como um processo que se dá diante de demandas ou eventos ambientais que são interpretados e

DOI: https://doi.org/10.25110/arqsaude.v19i1.2015.5265

${ }^{1}$ Enfermeira. Doutoranda do departamento de Enfermagem Psiquiátrica e Ciências Humanas da Escola de Enfermagem de Ribeirão Preto/USP. carlinhateixeira@hotmail.com

${ }^{2}$ Psicóloga. Mestranda em Psicologia da Faculdade de Filosofia, Ciências e Letras de Ribeirão Preto/USP. ericascrepaldi@gmail.com

${ }^{3}$ Enfermeira. Profa. Associada da Escola de Enfermagem de Ribeirão Preto. gherardidonato@gmail.com

${ }^{4}$ Enfermeira. Doutora em Enfermagem Psiquiátrica - Escola de Enfermagem de Ribeirão Preto/USP. mireisdorfer@gmail.com

${ }^{5}$ Psicóloga. Profa. Associada da Escola de Enfermagem de Ribeirão Preto. anacar@eerp.usp.br

${ }^{6}$ Psicóloga. Profa. Doutora da Faculdade de Medicina de Ribeirão Preto.plsantos@fmrp.usp.br

Endereço para correspondência: Carla Araujo Bastos Teixeira, Escola de Enfermagem de Ribeirão Preto - EERP/USP, Avenida dos Bandeirantes, 3900 Campus Universitário Sala 20, Bairro Monte Alegre CEP: 14040-902 Ribeirão Preto - SP. 
avaliados pelo indivíduo como desgastante ou que excedem os seus recursos colocando em risco o seu bem-estar (VANAELST et al., 2012).

Adotando então a perspectiva transacional do estresse, este se configura como o conjunto de reações físicas, psicológicas e sociais do organismo, visando à manutenção do seu equilíbrio, manifestadas diante da adaptação a um estressor. $\mathrm{O}$ estresse pode ser considerado patológico quando o indivíduo exaure seus recursos internos, levando-o ao sofrimento psíquico e a patologias ocasionadas por essa exaustão (CALDERERO et al., 2008).

A criança, assim como o adulto, está susceptível a situações percebidas como estressoras que, quando mantidas de forma contínua ou excessiva, podem ocasionar efeitos deletérios significativos no nível psicopatológico (EGELAND; KREUTER, 1991; MOMBELLI et al., 2011). O desenvolvimento do estresse se dá de forma atrelada às estratégias de enfrentamento adquiridas ao longo da vida do indivíduo, iniciadas principalmente no período da infância (LIPP, 2001). Por conseguinte, a investigação do estresse infantil é de extrema relevância para o provimento de intervenções efetivas no que tange à prevenção e ao tratamento do estresse em crianças e, consequentemente, na prevenção em adultos.

No Brasil, o estresse infantil começou a ser investigado no início da década de 1970, no estado de São Paulo. Tais estudos inferiram que havia diversas características comuns entre o estresse adulto e o estresse infantil (LIPP, 2000). A criança, durante seu desenvolvimento afetivo, emocional e intelectual, depara-se com diversas situações percebidas como estressoras, ou geradoras de tensão, com as quais muitas vezes ainda não possui habilidades para lidar. Entretanto, o aparecimento da sintomatologia do estresse não se reduz somente à associação do nível de tensão, tendo como geratriz eventos multifatoriais (LIPP, 2000; MOMBELLI et al., 2011).

A forma como têm sido conduzidos os estudos sobre estresse infantil podem trazer respostas no que tange às diversas perspectivas de como este conceito tem sido abordado pelos pesquisadores ao investigar a população infantil, aos desafios encontrados na mensuração deste constructo, bem como ao direcionamento de ações de intervenção. Há uma variedade de estratégias que vem sendo utilizadas para avaliação do estresse infantil, muitas delas utilizando combinações de instrumentos para avaliar eventos de vida e compará-los a respostas das crianças. Entretanto, não há uma sistematização a respeito de instrumentos para avaliação do estresse psicológico infantil, nem uma orientação sobre as vantagens e desvantagens das diferentes técnicas e estratégias. A fim de superar essa lacuna, objetivamos neste estudo identificar, a partir de uma revisão integrativa da literatura, quais testes psicológicos têm sido utilizados nos estudos que avaliam o estresse em crianças.

\section{Material e Método}

A fim de alcançar o objetivo proposto, definiu-se como método de pesquisa a revisão integrativa da literatura, pois ela permite reunir, avaliar de forma crítica e sintetizar os estudos realizados sobre o tema investigado, possibilitando uma conclusão a partir desses achados. A revisão integrativa permite a inclusão de estudos teóricos e empíricos e de abor- dagens metodológicas distintas (qualitativas e quantitativas). Os estudos selecionados para compor a revisão são analisados de forma sistemática com o objetivo de responder a pergunta norteadora da revisão de literatura e identificar possíveis lacunas que direcionam o desenvolvimento de pesquisas futuras (MENDES et al., 2008; POMPEO et al., 2009).

$\mathrm{O}$ método da revisão integrativa estabelece um processo bem definido constituído de seis etapas: (1) identificação do problema e elaboração da questão norteadora; (2) definição dos descritores para busca e dos critérios de inclusão e exclusão do estudo; (3) amostragem a partir do levantamento e seleção dos artigos; (4) leitura e avaliação crítica dos artigos incluídos na revisão; (5) extração de informações e preenchimento do banco de dados dos trabalhos revisados; (6) análise e discussão dos achados; e (7) síntese do conhecimento encontrado ou apresentação da revisão integrativa, propriamente dita (POMPEO et al., 2009).

A partir da questão "Quais são os testes psicológicos utilizados para avaliar estresse na infância?” foram identificados os descritores correspondentes às palavras-chave da pergunta, primeiramente em língua portuguesa por meio dos Descritores em Ciências da Saúde (DeCS) na Biblioteca Virtual em Saúde (BVS), e posteriormente os em língua inglesa no Medical Subject Headings (MeSH) na US National Library of Medicine. Foram identificados os termos "Estresse Psicológico", "Testes Psicológicos" e "Crianças" e "Psychological Stress", "Psychological Tests" e "Child". A busca de artigos foi realizada nas bases Lilacs, Ibecs, Medline, Biblioteca Cochrane e Scielo acessadas por meio da BVS e na PubMed, utilizando-se nos dois casos os três descritores ("Estresse Psicológico" AND "Testes Psicológicos" AND "Crianças" na BVS e "Psychological Stress" AND "Psychological Tests" AND “Child” na PubMed).

Os critérios de inclusão utilizados foram: idioma (inglês e português), ano de publicação (2004-2014), limite de idade (crianças até 12 anos) e tipo de documento (artigo original). Foram excluídos artigos de revisão, estudos com animais, estudos teóricos/ reflexivos, cartas ao editor, estudos que não estavam de acordo com os critérios de inclusão (que ainda apareceram após refinamento da busca). Em seguida, procedeu-se à leitura dos títulos dos artigos encontrados, sendo descartados aqueles que estavam em duplicada e que não contemplavam o objetivo do estudo. A posteriori, leu-se os resumos dos trabalhos encontrados em ambas as bases de dados para, em seguida, selecionar aqueles que abordavam os testes psicológicos utilizados em avaliação de estresse em crianças. Por fim, os artigos selecionados foram lidos na íntegra e três deles foram excluídos da amostra por não contemplar o objetivo desse estudo.

A busca ocorreu no mês de abril de 2014. Os artigos foram organizados em banco de dados de acordo com referência completa, país de origem dos autores e da revista, autores, objetivos, tipo do estudo, amostra, instrumento, testes psicológicos utilizados, procedimentos, principais resultados e referências secundárias. A partir da análise dos conteúdos dos estudos, identificaram-se três categorias temáticas principais, sendo elas: nível fisiológico, nível ambiental e nível psicológico. 
Resultados

As buscas na BVS e Pubmed evidenciaram 165 pu- blicações (Tabela 1), sobre as quais procedeu-se a sistematização da revisão integrativa, resultando em onze artigos para síntese do conteúdo.

Tabela 1: Distribuição dos artigos obtidos nas bases de dados segundo as etapas utilizadas para seleção dos artigos. Ribeirão Preto-SP, 2014.

\begin{tabular}{lcccccc}
\hline $\begin{array}{c}\text { Bases de } \\
\text { dados }\end{array}$ & $\begin{array}{c}\text { Artigos } \\
\text { localizados }\end{array}$ & $\begin{array}{c}\text { Artigos } \\
\text { selecionados } \\
\text { pela leitura } \\
\text { dos títulos }\end{array}$ & $\begin{array}{c}\text { Artigos } \\
\text { selecionados } \\
\text { pela leitura } \\
\text { dos resumos }\end{array}$ & $\begin{array}{c}\text { Artigos } \\
\text { disponíveis }\end{array}$ & $\begin{array}{c}\text { Artigos } \\
\text { selecionados } \\
\text { pela leitura na } \\
\text { íntegra }\end{array}$ & $\begin{array}{c}\text { Artigos } \\
\text { encontrados duas bases } \\
\text { nas }\end{array}$ \\
\hline BVS & 84 & 40 & 14 & 12 & 10 & 4 \\
Pubmed & 81 & 22 & 06 & 06 & 05 & 11 \\
Total & 165 & 62 & 20 & 18 & 15 & 11 \\
\hline
\end{tabular}

Após leitura e interpretação dos achados, os instrumentos utilizados para avaliar estresse nesses estudos foram divididos numa tabela de acordo com o tipo de abordagem do estresse: fisiológico, ambiental e psicológico (Quadro 1). O nível fisiológico engloba os marcadores biológicos, como o cortisol, por exemplo. O nível ambiental abrange as avaliações de estressores presentes em eventos de vida importantes, eventos diários e estressores crônicos. Já o nível psicológico corresponde às avaliações do indivíduo e às suas respostas emocionais diante de estressores, compreendendo desde os seus sentimentos de raiva até os estilos de coping.

Quadro 1: Distribuição dos testes piscológicos conforme nível de mensuração e utilização em cada estudo. Ribeirão Preto-SP, 2014

\begin{tabular}{|c|c|c|c|}
\hline Referência do estudo & Nível ambiental & Nível psicológico & Nível fisiológico \\
\hline $\begin{array}{l}\text { Vanaelst, B. et al. (2013) Cortisone in hair of } \\
\text { elementary school girls and its relationship with } \\
\text { childhood stress (VANAELST et al., 2013). }\end{array}$ & $\begin{array}{l}\text { - The Coddington Life } \\
\text { Events Scale (CLES) }\end{array}$ & $\begin{array}{l}\text { - The Coddington Life Events } \\
\text { Scale (CLES) } \\
\text { - Emoções básicas } \\
\text { - Estilos de coping }\end{array}$ & - Cortisol capilar \\
\hline $\begin{array}{l}\text { Rice, M. et al. (2008) Relationship of anger, } \\
\text { stress, and coping with school connectedness in } \\
\text { fourth-grade children (RICE et al., 2008). }\end{array}$ & $\begin{array}{l}\text { - Coping Resources In- } \\
\text { ventory Scales for Edu- } \\
\text { cational Enhancement } \\
\text { (CRISEE) - Subescala } \\
\text { Eventos estressantes } \\
\end{array}$ & $\begin{array}{l}\text {-Coping Resources Inventory } \\
\text { Scales for Educational Enhance- } \\
\text { ment (CRISEE) - Subescala Re- } \\
\text { cursos de coping percebidos }\end{array}$ & \\
\hline $\begin{array}{l}\text { Quesada, A. A. et al. (2012) Psychosocial stress } \\
\text { exposure impairs memory retrieval in children } \\
\text { (QUESADA et al., 2012). }\end{array}$ & & & $\begin{array}{l}\text {-Cortisol salivar } \\
\text {-Trier Social Stress } \\
\text { Test for Children - } \\
\text { TSST -C } \\
\end{array}$ \\
\hline $\begin{array}{l}\text { Lanni, K. E. et al. (2011) Verbal ability, social } \\
\text { stress, and anxiety in children with autistic di- } \\
\text { sorder (LANNI et al., 2012). }\end{array}$ & & & $\begin{array}{l}\text {-Cortisol salivar -Trier } \\
\text { Social Stress Test for } \\
\text { Children - TSST -C } \\
\end{array}$ \\
\hline $\begin{array}{l}\text { Wadsworth, M. E. et al. (2011) Testing the Adap- } \\
\text { tation to Poverty-Related Stress Model: Predic- } \\
\text { ting Psychopathology Symptoms in Families } \\
\text { Facing Economic Hardship (WADSWORTH et } \\
\text { al., 2011). }\end{array}$ & $\begin{array}{l}\text { - Multicultural Events } \\
\text { Schedule for Adolescents } \\
\text { (MESA) }\end{array}$ & $\begin{array}{l}\text { - Responses to Stress Question- } \\
\text { naire (RSQ) }\end{array}$ & \\
\hline $\begin{array}{l}\text { Kleiber e McCarthy (2006). Evaluating instru- } \\
\text { ments for a study on children's responses to a } \\
\text { painful procedure when parents are distraction } \\
\text { coaches (KLEIBER; MCCARTHY, 2006). }\end{array}$ & & $\begin{array}{l}\text { - Coping Behaviors Questionnai- } \\
\text { re (CBQ) } \\
\text { - Child Behavior Style Scale } \\
\text { (CBSS) }\end{array}$ & \\
\hline $\begin{array}{l}\text { Bernard, R. S. et al. (2004) Pediatric Procedural } \\
\text { Approach-Avoidance Coping and Distress: A } \\
\text { Multitrait-Multimethod Analysis (BERNARD } \\
\text { et al., 2004). }\end{array}$ & & $\begin{array}{l}\text { - Child Approach-Avoidance Ra- } \\
\text { ting Scale (CAARS) } \\
\text { - BAADS } \\
\text { - Approach- Avoidance Behavior } \\
\text { Scale (AABS) }\end{array}$ & \\
\hline
\end{tabular}




\begin{tabular}{|l|l|l|l|}
\hline $\begin{array}{l}\text { De Veld, M. J. et al. (2012). The relation betwe- } \\
\text { en emotion regulation strategies and physiolo- } \\
\text { gical stress responses in middle childhood. (DE } \\
\text { VELD et al., 2012) }\end{array}$ & $\begin{array}{l}\text { - Emotion Regulation Question- } \\
\text { naire (ERQ) }\end{array}$ & $\begin{array}{l}\text { - Cortisol salivar } \\
- \text { Trier Social Stress } \\
\text { Test for Children - } \\
\text { TSST-C }\end{array}$ \\
\hline $\begin{array}{l}\text { Montirosso, R. et al. (2010) Infant's emotional } \\
\text { variability associated to interactive stressful si- } \\
\text { tuation: A novel analysis approach with Sample } \\
\text { Entropy and Lempel. (MONTIROSSO et al., } \\
\text { 2010) }\end{array}$ & & $\begin{array}{l}\text { Face-to-face and still-face inte- } \\
\text { ractions (FFSF) }\end{array}$ & \\
\hline $\begin{array}{l}\text { Sayfan, L. et al. (2008). Children's expres- } \\
\text { sed emotions when disclosing maltreatment. } \\
\text { (SAYFAN et al., 2008) }\end{array}$ & & $\begin{array}{l}\text { Child Version (TSC-C) } \\
- \text { Post-Traumatic Symptom In- } \\
\text { ventory for Children (PT-SIC) }\end{array}$ & \\
\hline $\begin{array}{l}\text { Lohaus, A. et al (2004). The prediction of heal- } \\
\text { th-related behaviour in elementary school chil- } \\
\text { dren. (LOHAUS et al., 2004) }\end{array}$ & $\begin{array}{lll}\text {-Youth Self Report } \\
\text { (YSR) Youth Self Report (YSR) } \\
\text { - Coping Behavior }\end{array}$ & \\
\hline
\end{tabular}

Os estudos da amostra apresentaram estratégias diversas para mensurar o estresse infantil que são expressas no uso de diferentes testes psicológicos, instrumentos ou combinações distintas entre estes. Dentre os onze artigos que compuseram a amostra dessa revisão, o estresse foi avaliado por meio do nível ambiental em quatro estudos, pelo nível psicológico em dezesseis estudos e pelo nível fisiológico em quatro estudos.

Os níveis ambiental e psicológico foram investigados mediante questionários e entrevistas. Dentre esses instrumentos três são respondidos pelos pais e doze pelas crianças. Dois estudos utilizaram o relato dos pais: um utilizou somente relatos dos pais e outro utilizou um instrumento de relato dos pais e outro de autorelato das crianças. Além disso, três dos instrumentos abrangem ambos os níveis psicológico e ambiental: The Coddington Life Events Scale (CLES), Coping Resources Inventory Scales for Educational Enhancement (CRISEE) e o Youth Self Report (YSR).

O nível fisiológico foi avaliado por meio da mensuração do cortisol capilar e salivar. A medida de cortisol capilar foi associada em um estudo a medidas nos níveis ambientais e psicológicos e em outros três estudos foi medido o cortisol salivar combinado a um protocolo - Trier Social Stress Test for Children (TSST-C) (KIRSCHBAUM; PIRKE; HELLHAMMER, 1993). Este protocolo compreende uma manipulação que induz uma situação experimental de estresse.

Com exceção dos marcadores biológicos, dezoito instrumentos diferentes foram utilizados para avaliar o estresse. Destes, doze tiveram suas qualidades psicométricas apresentadas, doze foram referenciados apropriadamente e cinco foram desenvolvidos ou adaptados para atender os objetivos do estudo.

\section{Discussão}

A presente pesquisa teve como objetivo identificar os testes psicológicos que avaliam o estresse psicológico em crianças, a partir de um levantamento de artigos indexados por descritores referentes a esses assuntos. Como resultado obteve-se diversas estratégias de avaliação do estresse que se diferenciam dos testes psicológicos, ou seja, que não atendem os critérios de padronização, validação e apresentação de índices de qualidades psicométricas consistentes.
É possível que os resultados apontem um direcionamento controverso nas pesquisas publicadas, pois os resultados obtidos, em uma busca prévia exploratória sobre testes psicológicos que avaliam o estresse infantil por meio do uso da palavra-chave "estresse" identificou-se que haviam estudos que avaliam estresse da criança indexados por descritores aleatórios (diversos), como "estresse fisiológico", "ansiedade" e "saúde mental". Com o intuito de manter a sistematização proposta com rigor analítico, incluiu-se nas buscas somente descritores contidos no DeCS e MeSH, descritos na metodologia, excluindo palavras-chaves com sentido mais amplo. A escolha por este método teve como objetivo tornar o estudo o mais consistente possível, indo ao encontro do preconizado pelos periódicos de renome científico da área da saúde.

Alguns testes psicológicos destinados a outras faixas etárias, como o Youth Self Report (YSR) destinado a adolescentes, foram adaptados para a aplicação em crianças. Outros instrumentos apresentados como testes psicológicos foram elaborados especificamente para a finalidade do estudo. Algumas dessas pesquisas não apresentaram informações as qualidades psicométricas de seus instrumentos, o que as tornam frágeis, limitando a confiabilidade e a generalização de seus dados. A ausência de testes psicológicos destinados a crianças nestes estudos pode sinalizar a escassez de instrumentos destinados a esta faixa etária (VANAELST et al., 2012).

A deficiência de testes psicológicos que avaliam estresse infantil também aponta a dificuldade de mensurá-lo, primeiramente porque o estresse é um processo complexo, de aferição multicentralizada, e segundo porque o autorelato da criança ainda tem sua credibilidade questionada. Vanaelst et al. (2012) aponta que embora os autorelatos de crianças apresentem boa confiabilidade a partir dos seus quatro anos, aumentando-a com a idade, é frequente pesquisadores utilizarem o relato dos pais, principalmente o da mãe, para avaliar as experiências emocionais da criança (VANAELST et al., 2012).

Contudo, os estudos abordados por esta revisão mostraram a predominância de autorelatos da criança, estando presente em apenas um estudo o uso exclusivo de relatos dos pais. A escolha entre o relato dos pais ou o autorelato de suas crianças foi discutida no estudo realizado por Lagattuta e colaboradores (2012), o qual comparou o relato dos pais 
com o dos seus filhos sobre avaliação dos sentimentos da criança (LAGATTUTA,SAYFAN; BAMFORD, 2012). Apesar de não ter sido possível concluir qual é o relato mais preciso, identificaram que os pais subestimam a frequência com que seus filhos se sentem ansiosos ou preocupados e superestimar os pensamentos e sentimentos otimistas de seus filhos. Constataram também que os pais parecem ter dificuldade em separar a sua perspectiva emocional da de seus filhos. Portanto, é importante que se obtenha o relato de múltiplos informantes, como os dos pais, dos professores e o da própria criança.

A abrangência de avaliações do estresse para além do nível psicológico expressa também a concepção do estresse numa perspectiva transacional. A catalogação dos instrumentos nos níveis fisiológico, ambiental e psicológico utilizada neste artigo vai ao encontro da literatura que entende o processo de estresse como uma maneira do indivíduo interpretar e avaliar as demandas ou os eventos ambientais como desgastante ou que excedem os seus recursos, ameaçando o seu bem-estar. Este processo compreende três componentes distintos: a fonte de estresse ou estressores, a avaliação e a resposta afetiva do indivíduo aos estressores e, por fim, a resposta de estresse fisiológico (VANAELST et al., 2012).

\section{Conclusão}

Diante do exposto, percebe-se que independente da corrente conceitual ou da teoria psicológica que embase o estresse, condições estressantes podem ser desencadeadoras de malefícios na saúde como um todo. Dessa forma, entender como as pesquisas estão caminhando no sentido de abranger uma população tão peculiar como a infantil, faz necessário a fim de prevenir adoecimento biopsiquico o mais precoce possível.

A partir da apropriação desse saber, o estabelecimento de relações entre os testes psicológicos utilizados na mensuração do estresse infantil e a forma como o estresse em crianças vem sendo abordado (nível fisiológico, psicológico ou ambiental) fornece um posicionamento quanto à abordagem dos profissionais que trabalham com a clientela descrita. Assim, o conhecimento desse panorama faz-se primordial para o avanço de pesquisas na temática, bem como direcionamento para profissionais da aérea assistencialista.

Considera-se, portanto, relevante o desenvolvimento de estudos que elucidem a avaliação do estresse em crianças para que, deste modo, haja contribuição na formação de um panorama situacional que subsidie reflexões acerca destes fenômenos colaborando assim com o desenvolvimento de pesquisas neste campo de estudo.

\section{Referências}

BERNARD, R. S. et al. Pediatric procedural approachavoidance coping and distress: a multitrait-multimethod analysis. J Pediatr Psychol, v. 29, n. 2, p. 131-41, Mar 2004.

CALDERERO, A. R. L.; MIASSO, A.; CORRADIWEBSTER, C. M. Estresse e estratégias de enfrentamento em uma equipe de enfermagem de Pronto Atendimento.

Rev. Eletr. Enf. (Internet), v. 10, n. 1, p. 51-62, 2008.
DE VELD, D. M.; RIKSEN-WALRAVEN, J. M.; DE WEERTH, C. The relation between emotion regulation strategies and physiological stress responses in middle childhood. Psychoneuroendocrinology, v. 37, n. 8, p. 1309-19, Aug 2012.

EGELAND, B.; KREUTER, C. Maternal stress on high-risk children. In: CUMMINGS, E. M.;GREENE, A. L., et al. (Ed.). Life-Span developmental psychology: perspectives on stress an coping. Hillsdale, New Jersey: Lawrence Erlhaum Associates Publishers, 1991.

GHERARDI-DONATO, E. C. Estresse ocupacional, estresse precoce e estratégias de enfrentamento entre auxiliares e técnicos de enfermagem de um hospital universitário. 2013. Livre Docência Enfermagem Psiquiátrica, Escola de Enfermagem de Ribeirão Preto Universidade de São Paulo, Ribeirão Preto.

HOLMES, T.; RAHE, R. The Social Readjustment Rating Scale. Journal of Psychosomatic Research, v. 11, p. 5, 1967.

KIRSCHBAUM, C.; PIRKE, K. M.; HELLHAMMER, D. $H$. The 'Trier Social Stress Test'--a tool for investigating psychobiological stress responses in a laboratory setting. Neuropsychobiology, v. 28, n. 1-2, p. 76-81, 1993.

KLEIBER, C.; MCCARTHY, A. M. Evaluating instruments for a study on children's responses to a painful procedure when parents are distraction coaches. J Pediatr Nurs, v. 21, n. 2, p. 99-107, 2006.

LAGATTUTA, K. H.; SAYFAN, L.; BAMFORD, C. Do you know how I feel? Parents underestimate worry and overestimate optimism compared to child self-report. J Exp Child Psychol, v. 113, n. 2, p. 211-32, 2012.

LANNI, K. E. et al. Verbal ability, social stress, and anxiety in children with autistic disorder. Autism, v. 16, n. 2, p. 123-38, 2012.

\section{LAZARUS, R.; FOLKMAN, S. Stress appraisal and} coping. New York: Springer Publishing Company, 1984.

LIPP, M. E. N. O estresse da criança e suas consequências. In: LIPP, M. E. N. (Ed.). Criança estressada: causas, sintomas e soluções. Campinas: Papirus, 2000.

. Estresse emocional: a contribuição de estressores internos e externos. . Rev. Psiq. Clín., v. 28, n. 6, p. 2, 2001.

LOHAUS, A. et al. The prediction of health-related behaviour in elementary school children. J Health Psychol, v. 9, n. 3, p. 375-9, 2004.

MENDES, K. D. S.; SILVEIRA, R. C. D. C. P.; GALVÃO, C. M. Revisão integrativa: método de pesquisa para a incorporação de evidências na saúde e na enfermagem.

Texto \& Contexto - Enfermagem, v. 17, p. 758-764, 2008. 
MOMBELLI, M. A. et al. Estrutura e suporte familiar como fatores de risco de stress infantil. Estudos de Psicologia (Campinas), v. 28, p. 327-335, 2011.

MONTIROSSO, R. et al. Infant's emotional variability associated to interactive stressful situation: a novel analysis approach with Sample Entropy and Lempel-Ziv Complexity. Infant Behav Dev, v. 33, n. 3, p. 346-56, 2010.

NAKASATO, H. K.; CAROMANO, F. A. Estresse - Os

Fundamentos Necessários para Compreensão das Alterações Clínico-Funcionais. Arquivos de Ciências da Saúde da

UNIPAR, v. 4, n. 3, p. 6, 2000.

POMPEO, D. A.; ROSSI, L. A.; GALVÃO, C. M.

Revisão integrativa: etapa inicial do processo de validação de diagnóstico de enfermagem. Acta Paulista de Enfermagem, v. 22, p. 434-438, 2009.

QUESADA, A. A. et al. Psychosocial stress exposure impairs memory retrieval in children.

Psychoneuroendocrinology, v. 37, n. 1, p. 125-36, 2012.

RICE, M. et al. Relationship of anger, stress, and coping with school connectedness in fourth-grade children. J Sch Health, v. 78, n. 3, p. 149-56, 2008.

SAYFAN, L. et al. Children's expressed emotions when disclosing maltreatment. Child Abuse Negl, v. 32, n. 11, p. 1026-36, 2008.

SELYE, H. The stress of life. New York: McGraw-Hill, 1956.

TEIXEIRA, C. A. B. Estresse ocupacional e estratégias de enfrentamento entre profissionais de enfermagem em ambiente hospitalar. 2013. Dissertation (Mestrado). Enfermagem Psiquiátrica, Escola de Enfermagem de Ribeirão Preto - Universidade de São Paulo, Ribeirão Preto.

VANAELST, B. et al. Epidemiological approaches to measure childhood stress. Paediatr Perinat Epidemiol, v. 26, n. 3, p. 280-97, 2012.

Cortisone in hair of elementary school girls and its relationship with childhood stress. Eur J Pediatr, v. 172, n. 6, p. 843-6, 2013.

WADSWORTH, M. E. et al. Testing the adaptation to poverty-related stress model: predicting psychopathology symptoms in families facing economic hardship. J Clin Child Adolesc Psychol, v. 40, n. 4, p. 646-57, 2011. 